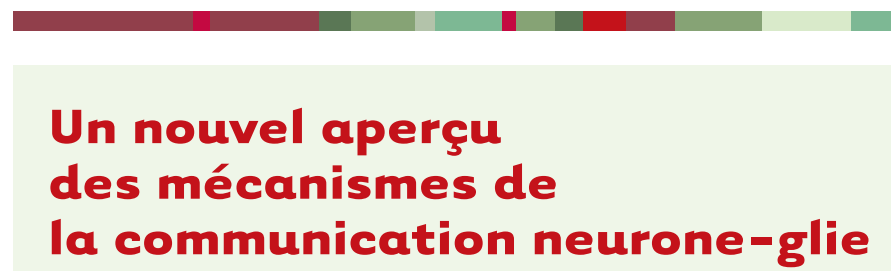

Frank W. Pfrieger, Michael Reber
CNRS UPR 3212, université de Strasbourg, institut des neurosciences cellulaires et intégratives ( $\mathrm{INCl}$ ),

5, rue Blaise Pascal, 67084 Strasbourg, France.

fw-pfrieger@gmx.de

frank.pfrieger@inci-cnrs.unistra.fr
Le fonctionnement du cerveau repose sur la communication entre neurones et cellules gliales

Chacun de nos organes fonctionne grâce à la communication entre les cellules qui le composent. Les neurones, cellules clés du cerveau, utilisent un mode de communication très sophistiqué via des structures spécialisées, les synapses chimiques. Ces synapses permettent la transmission de l'information nerveuse en convertissant le signal électrique qui se propage le long de l'axone en signal chimique, via l'émission de neurotransmetteurs, par le neurone présynaptique, dans la fente synaptique. Ces neurotransmetteurs vont activer le neurone postsynaptique, transmettant ainsi l'information. La libération des neurotransmetteurs s'effectue par un mécanisme d'exocytose. Au cours de ce processus, une augmentation transitoire de calcium dans le neurone présynaptique provoque la fusion de vésicules contenant le neurotransmetteur avec la membrane plasmique via le complexe protéique SNARE (soluble $\mathrm{N}$-ethylmaleimide-sensitive factor attachment protein receptor), complexe hautement sensible à la toxine botulique [1]. Outre les neurones, le système nerveux contient également des cellules non neuronales, baptisées neuroglie (colle neuronale) par le pathologiste allemand Rudolf Virchow en 1856. La neuroglie remplit l'espace interneuronal et fournit un support structurel et métabolique aux neurones [2]. Différents types cellulaires, dont les astrocytes, la glie de Bergmann dans le cervelet et les cellules de Müller dans la rétine, enrobent les synapses du système nerveux central. Plusieurs résultats expérimentaux indiquent que les cellules gliales détectent l'activité synaptique et régulent la transmission synaptique via la libération de gliotransmetteurs (notamment le glutamate, un transmetteur excitateur) selon un mécanisme encore inconnu. Une hypothèse suggère que la libération de ces gliotransmetteurs reposerait

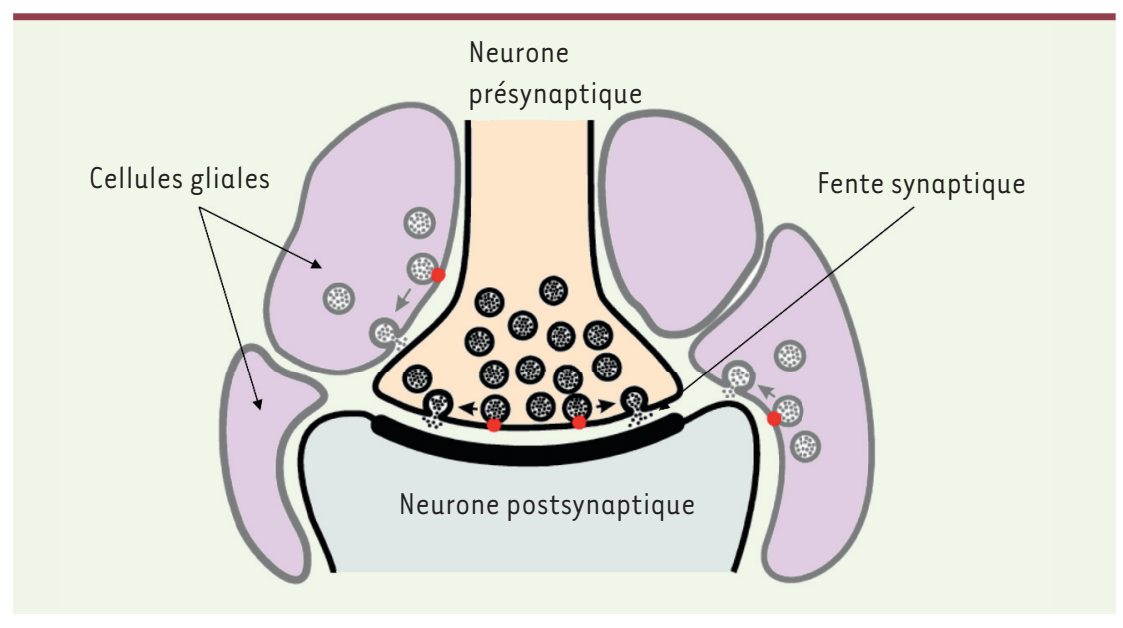

sur un mécanisme d'exocytose induite par le calcium, similaire à celui qu'utilisent les neurones pour la libération des neurotransmetteurs au niveau de la synapse [3] (Figure 1).

Rôle de l'exocytose dépendante du calcium dans les cellules gliales de la rétine

En 2004, nous avons décidé d'élaborer un nouvel outil expérimental permettant de comprendre le rôle physiologique de l'exocytose induite par le calcium dans les cellules gliales. Cet outil, la souris iBot (pour induced botulin toxin), permet l'expression de la chaîne légère de la neurotoxine botulique de sérotype B (BoNT/B) dans des cellules d'intérêt [4]. L'activité de cette enzyme, issue de Clostridium botulinum, détruit les composants du complexe SNARE, inhibant l'exocytose induite [5]. Ce modèle a été validé en démontrant que l'expression de BoNT/B dans l'organisme entier est mortelle, et que sa présence dans les neurones réduit fortement la transmission synaptique (Figure 2).

Afin de déterminer le rôle physiologique de l'exocytose dans les cellules gliales, nous avons choisi la rétine, tissu nerveux qui tapisse le fond de l'œil et transforme les stimulus visuels en signaux électriques transmis au cerveau.

Figure 1. Gliotransmission et neurotransmission. Ce diagramme illustre l'hypothèse selon laquelle les cellules gliales libèrent des substances dans la fente synaptique par le même mécanisme que les neurones, une exocytose dépendante du calcium (cercles rouges). 

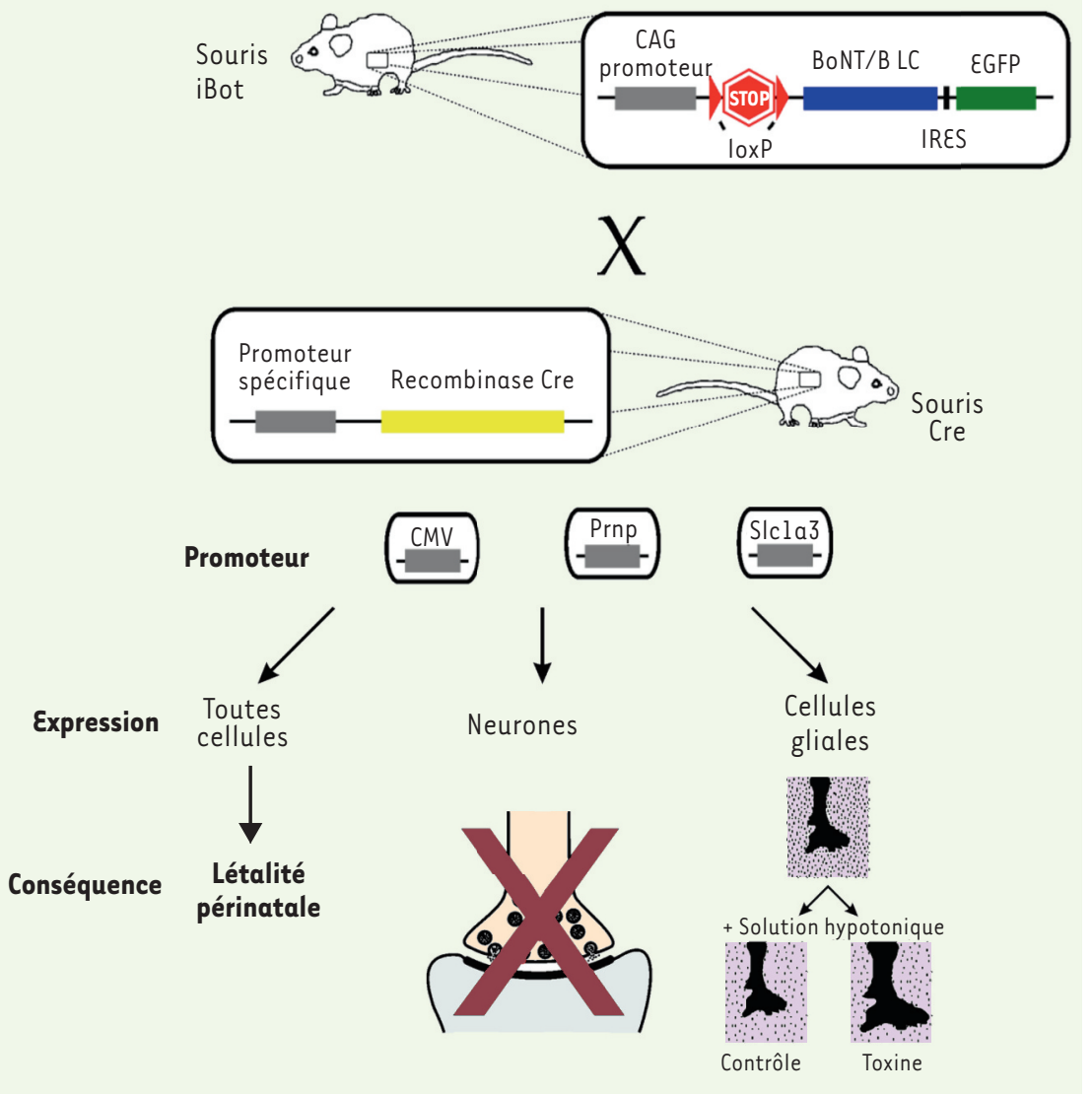

Figure 2. Analyse de l'exocytose induite par le calcium dans la souris en utilisant le système Cre/loxP. La souris iBot porte le gène codant pour la toxine botulique (BoNT/B) et un marqueur fluorescent. Ces souris sont croisées avec une lignée exprimant la recombinase Cre. La toxine et le marqueur fluorescent sont exprimés dans les cellules où l'enzyme Cre excise une séquence STOP flanquée de sites de reconnaissance (IoxP). La spécificité cellulaire est définie par le type de promoteur contrôlant l'expression de la recombinase Cre. Trois différentes lignées Cre sont présentées. L'expression ubiquitaire de la toxine, induite par la recombinase sous le contrôle des éléments de régulation du cytomégalovirus (CMV), provoque une létalité périnatale chez la souris. L'expression neuronale de la toxine, induite lorsque la recombinase est sous le contrôle des éléments de régulation de la protéine du prion (Prnp), conduit à une inhibition de la transmission synaptique. Enfin, l'expression gliale de la toxine, induite lorsque la recombinase est sous le contrôle des éléments de régulation de Slcla3, provoque des dysfonctionnements de régulation du volume cellulaire dans les cellules gliales de Müller.

Ce modèle présente de nombreux avantages: sa physiologie et sa morphologie peuvent être examinées sur l'animal vivant. Sa structure laminée, où la position des différents types cellulaires et leurs synapses est parfaitement connue, simplifie l'analyse ultrastructurale et histologique. Enfin, la rétine ne contient qu'un type de cellules gliales, les cellules de Müller, découvertes par l'anatomiste allemand Heinrich Müller (cinq années avant Virchow) et identifiées comme des fibres radiaires ${ }^{1}[6]$. Ces cellules occupent l'entière épaisseur de la rétine

\footnotetext{
${ }^{11}$ En 1853, Müller et son collègue Kölliker écrivèrent à l'Académie des sciences : «Un des faits le plus digne d'attention, c'est que, d'après les observations de l'un de nous (Müller) sur les animaux, confirmées par nous pour la rétine humaine, il provient de la partie interne de chaque cône et de chaque bâtonnet une fibre qui, après avoir traversé toutes les couches de la rétine, va se perdre à la face interne de la membrane limitante. Ces fibres [...] forment un système tout à fait particulier de la rétine, et ont été nommées par nous les fibres radiaires. » [6].
}

et sont au contact de l'ensemble des neurones et de leurs synapses [7]. Le croisement de la lignée iBot avec une lignée permettant de cibler spécifiquement les cellules gliales [8] a permis d'induire l'expression de BoNT/B dans les cellules de Müller. La mise au point d'un nouveau test biochimique nous a permis de démontrer la libération de glutamate par les cellules de Müller après stimulation. Cette libération de glutamate est diminuée en présence de toxine, sans que la structure morphologique de la rétine soit affectée, comme le montrent des données d'imagerie in vivo, d'histologie et d'ultrastructure. De plus, la fonction rétinienne, caractérisée par des méthodes électrophysiologiques et comportementales chez l'animal, reste intacte en présence de toxine botulique. En revanche, nous avons identifié un défaut de régulation du volume des cellules de Müller, confirmant les hypothèses proposées par le groupe de Andreas Reichenbach (Paul Flechsig institute for brain research, Leipzig, Allemagne).

\section{Conclusions et perspectives}

Nos travaux ont permis de clarifier le rôle physiologique de l'exocytose des cellules gliales dans la rétine, ouvrant de nouvelles voies de recherche. La preuve que le glutamate peut être libéré par différents mécanismes, dont l'exocytose, suscite un intérêt particulier pour l'étude de ces mécanismes dans d'autres de types de cellules gliales. La conservation de la physiologie intacte de la rétine en présence de toxine suggère que d'autres mécanismes de libération des gliotransmetteurs existent, dont l'étude nécessitera la mise au point de nouveaux modèles expérimentaux. D'autre part, l'abolition de l'exocytose gliale peut affecter des fonctions cérébrales non étudiées dans ce travail. En parallèle de notre étude, un laboratoire américain a développé un modèle murin permettant l'étude du rôle de l'exocytose gliale in vivo [9]. Dans cette étude, les souris ne 
présentent pas de défauts cognitifs ou de coordination motrice, mais des anomalies de l'homéostasie du sommeil. En conclusion, nos travaux démontrent la participation de l'exocytose des cellules gliales à des fonctions nerveuses spécifiques et soulignent l'importance du développement, parfois laborieux, de nouveaux modèles expérimentaux. Notre lignée de souris iBot représente un outil d'étude du rôle de l'exocytose dans divers types cellulaires et permet l'analyse de la communication intercellulaire au sein de différents organes, dont le cerveau. $\diamond$

Cellular communication between neurons and glial cells: an overview

\section{REMERCIEMENTS}

Nous remercions nos collègues qui ont participé à cette étude et présentons nos excuses à ceux dont les travaux n'ont pu être cités faute d'espace suffisant.

\section{LIENS D'INTÉRÊT}

Les auteurs déclarent n'avoir aucun lien d'intérêt concernant les données publiées dans cet article.

\section{RÉFÉRENCES}

1. Sudhof TC, Rizo J. Synaptic vesicle exocytosis. Cold Spring Harb Perspect Biol 2011 ; 3 : doi:pii: a005637. 10.1101/cshperspect.a005637.

2. Kettenmann H, Ransom BR. Neuroglia. New York : Oxford University Press, 2004.

3. Zorec R, Araque A, Carmignoto G, et al. Astroglial excitability and gliotransmission: an appraisal of $\mathrm{Ca} 2+$ as a signalling route. ASN Neuro $2012 ; 4$ : doi:pii: e00080. 10.1042/AN20110061.
4. Slezak M, Grosche A, Niemiec A, et al. Relevance of exocytotic glutamate release from retinal glia. Neuron $2012 ; 74$ : 504-16.

5. Poulain B. Botulinum neurotoxin. Rev Neurol (Paris) $2010 ; 166: 7-20$.

6. Kölliker A, Müller J. Compte Rendu hebdomadaires des Séances de l'Académie des Sciences 1853 ; 37 : 488 92.

7. Bringmann A, Pannicke T, Grosche J, et al. Muller cells in the healthy and diseased retina. Prog Retin Eye Res $2006 ; 25: 397-424$.

8. Slezak M, Goritz C, Niemiec A, et al. Transgenic mice for conditional gene manipulation in astroglial cells. GLIA 2007 ; 55 : 1565-76.

9. Halassa MM, Florian C, Fellin T, et al. Astrocytic modulation of sleep homeostasis and cognitive consequences of sleep loss. Neuron 2009 ; 61 : 213-9.

\section{NOUVELLE}

\section{Un nouveau concept de mécanisme de clairance respiratoire?}

Camille Ehré

\author{
Cystic fibrosis Center/Pulmonary medicine, \\ University of North Carolina at Chapel Hill, \\ 7027 Thurston Bowles BIdg, Chapel Hill, NC, 27599, \\ États-Unis. \\ camille_ehre@med.unc.edu
}

$>$ Dans toutes les muqueuses, respiratoire, gastro-intestinale ou encore celle des voies génitales, la présence d'un film viscoélastique de mucus est nécessaire pour protéger l'organisme contre l'invasion d'agents pathogènes, qu'il s'agisse de virus, bactéries ou autres polluants. Ce mucus est constitué principalement de larges glycoprotéines, appelées mucines, qui doivent être parfaitement hydratées pour maintenir les propriétés viscoélastiques du mucus. Dans les voies respiratoires, la couche de mucus séquestre les agents inhalés et progresse, grâce aux battements ciliaires des cellules sous-jacentes, vers la glotte. Le mucus est continuellement avalé ou expectoré, ce qui définit le mécanisme de clairance pulmonaire. Depuis des décennies, le dogme veut que les cellules ciliées battent librement dans un milieu aqueux et propulsent le mucus qui flotte sur l'épithélium respiratoire, mais nous avons récemment réfuté ce concept dans une étude publiée dans Science [1]. En effet, l'ancienne notion ne permet pas d'expliquer l'incidence des plaques de mucus observées dans les maladies caractérisées par l'obstruction pulmonaire (par exemple: bronchite chronique, mucoviscidose ou asthme). Notre étude montre que l'espace périciliaire est en fait occupé par de larges glycoprotéines organisées de manière spécifique en un réseau ayant une densité supérieure à celle de la couche mobile de mucus sus-jacente. Ce réseau dense de macromolécules est attaché aux cellules ciliées et possède les mêmes propriétés qu'un gel.
Le milieu périciliaire est un gel et non pas un fluide

Le concept erroné d'un milieu périciliaire fluide repose sur plusieurs données: des observations histopathologiques acquises avec des techniques anciennes et montrant un milieu cristallin, dépourvu de toute structure, mais aussi la perception intuitive qu'un milieu liquide serait plus adapté aux battements ciliaires. Cependant une analyse détaillée des propriétés biophysiques de polymères révèle que ce système de clairance pulmonaire ne pourrait pas être fonctionnel sans la présence de macromolécules capables de retenir l'eau occupant l'espace périciliaire. En l'absence de telles molécules, la couche de mucus, qui exerce une certaine pression osmotique, entraînerait l'assèchement du milieu 